Astrophysical Journal, ACCEPTED FeBruary 2004

Preprint typeset using LATEX style emulateapj v. 11/12/01

\title{
A SUB-MILLIMETER SEARCH OF NEARBY YOUNG STARS FOR COLD DUST: DISCOVERY OF DEBRIS DISKS AROUND TWO LOW-MASS STARS
}

\author{
Michael C. Liu, ${ }^{1}$ Brenda C. Matthews, ${ }^{2,3}$ Jonathan P. Williams, ${ }^{1}$ Paul G. Kalas ${ }^{2}$ \\ Astrophysical Journal, accepted February 2004
}

\begin{abstract}
We present results from a JCMT/SCUBA $850 \mu \mathrm{m}$ search for cold dust around eight nearby young stars belonging to the $\beta$ Pic $(t \approx 12 \mathrm{Myr})$ and the Local Association $(t \approx 50 \mathrm{Myr})$ moving groups. Unlike most past sub-mm studies, our sample was chosen solely on the basis of stellar age. Our observations achieve about an order of magnitude greater sensitivity in dust mass compared to previous work in this age range. We detected two of the three $\mathrm{M}$ dwarfs in our sample at $850 \mu \mathrm{m}$, GJ 182 and GJ $803\left(M_{\star} \approx 0.5 M_{\odot}\right)$, with inferred dust masses of only $\approx 0.01-0.03 M_{\oplus}$. GJ 182 may also possess a $25 \mu \mathrm{m}$ excess, indicative of warm dust in the inner few AU of its disk. For GJ 803 (AU Mic; HD 197481), sub-mm mapping finds that the $850 \mu \mathrm{m}$ emission is unresolved. A non-detection of the CO 3-2 line indicates the system is gas-poor, and the spectral energy distribution suggests the presence of a large inner disk hole $(\approx 17 \mathrm{AU}$ $=1.7^{\prime \prime}$ in radius for blackbody grains). These are possible indications that planets at large separations can form around M dwarfs within 10 Myr. In a companion paper (Kalas, Liu \& Matthews 2004), we confirm the existence of a dust disk around GJ 803 using optical coronagraphic imaging. Given its youthfulness, proximity, and detectability, the GJ 803 disk will be a valuable system for studying disk, and perhaps planet, formation in great detail. Overall, sub-mm measurements of debris disks point to a drop in dust mass by a factor of $\sim 10^{3}$ within the first $\sim 10 \mathrm{Myr}$, with the subsequent decline in the masses of sub-mm detected disks consistent with $t^{-0.5}$ to $t^{-1}$.
\end{abstract}

Subject headings: circumstellar matter — planetary systems: formation — planetary systems: protoplanetary disks - stars: late-type

\section{INTRODUCTION}

Circumstellar disks are common around stars at ages of a few million years. Rotationally supported disks are a natural outcome of the star formation process, because of the need to conserve angular momentum during the collapse of the natal molecular core. In addition, disks are central to current theories of planet formation. These young disks are composed of gas and dust, with estimated masses as large as several percent of the stellar mass. By virtue of their large optical depths, they can be easily detected and have been intensively studied.

Over the span of $\sim 5-10 \mathrm{Myr}$, these primordial disks are thought to dissipate (e.g. Strom et al. 1993; Haisch et al. 2001b), based on the observed decline of IR excesses, which originate from warm dust in the inner disk. At older ages, planetesimals and planets comprise the remaining circumstellar population. Collisions between the planetesimals produce dust grains, which form a "second-generation" disk. The Infrared Astronomical Satellite (IRAS) discovered the first example of a such dusty debris disk around Vega (Aumann et al. 1984). Overall, volume-limited farIR surveys have found that about $15 \%$ of main-sequence stars have debris disks (Backman \& Paresce 1993; FajardoAcosta et al. 1999; Gaidos 1999; Plets \& Vynckier 1999; Habing et al. 2001; Laureijs et al. 2002). Such disks have a small amount of dust, little evidence for gas, low characteristic temperatures indicative of grains at tens of $\mathrm{AU}$ separations, and a dearth of warm material in their inner regions (e.g., Zuckerman 2001). These attributes make debris disks physically distinct from primordial disks and lead to much weaker observational signatures.

Loss processes for the dust in debris disks act quickly compared to the age of the host stars, and the debris must be continually replenished. Thus, studying debris disks over a wide range of ages can provide insight into their evolution and physical origin. Most studies have focused on stars with ages of $\gtrsim 200 \mathrm{Myr}$ (Habing et al. 1999, 2001; Spangler et al. 2001; Laureijs et al. 2002), a reflection of the fact that most nearby stars are old.

The last few years have seen the discovery of many stars which are both young $(t \approx 10-100 \mathrm{Myr})$ and close to Earth $(d \lesssim 100 \mathrm{pc})$. These stars have largely been found in kinematically associated moving groups, by combining accurate space motions with evidence for youthfulness (e.g., Li abundances, X-ray emission, and chromospheric activity). They include the $\beta$ Pic $(\approx 8-20 \mathrm{Myr}$; Zuckerman et al. 2001a) and the Local Association $(\approx 20-150$ Myr; Jeffries 1995; Montes et al. 2001) moving groups. These are important systems for disk studies as they span an age range which is poorly studied. In addition, this timescale is associated with assemblage of terrestrial planets in our own Solar System (e.g. Wadhwa \& Russell 2000), so understanding disk properties at this epoch can illuminate the overall physical context for planet formation.

Given the cold characteristic temperatures of the dust $(\approx 40-100 \mathrm{~K}$; e.g., Dent et al. 2000), sub-mm continuum observations of debris disks are valuable. The emission is optically thin and hence can be used to estimate the disk masses, with the caveat that large bodies are missed. Previous sub-mm studies have focused on stars already known

${ }^{1}$ Institute for Astronomy, University of Hawai'i, 2680 Woodlawn Drive, Honolulu, HI 96822

2 Astronomy Department, University of California, Berkeley, CA 94720

${ }^{3}$ Radio Astronomy Laboratory, University of California, Berkeley, CA 94720 
Liu et al.

to have strong IR excesses (Zuckerman \& Becklin 1993; Sylvester et al. 1996; Holland et al. 1998; Greaves et al. 1998; Sylvester \& Mannings 2000; Sylvester et al. 2001; Holland et al. 2003; Holmes et al. 2003), with the most outstanding examples being spatially resolved at sub-mm wavelengths ( $\beta$ Pic, $\epsilon$ Eri, Fomalhaut, Vega).

Much less has been done in the way of "blind" searches of stars in a given age range to understand their sub-mm disk frequency and properties. Wyatt et al. (2003) have recently surveyed the secondary stars of binaries with $\mathrm{O}$ and B-type primaries, a sample originally identified by Lindroos (1986). By virtue of the primary stars being on the main sequence, the systems are $\lesssim 100$ Myr old. Previous searches for dust around this sample had relatively poor sensitivity (Gahm et al. 1994; Jewitt 1994; Ray et al. 1995). Of particular interest is the suggestion by Wyatt et al. that some stars may harbor very cold $(\lesssim 40 \mathrm{~K})$ disks, which would be missed by previous far-IR searches and only detected in the sub-mm. However, one limitation of the Lindroos sample is that most of the systems are fairly distant, which impacts the resulting disk sensitivity of sub-mm observations.

In this Letter, we present an $850 \mu \mathrm{m}$ search for cold dust around young stars in the $\beta$ Pic and Local Association moving groups. Unlike most previous sub-mm studies, our sample was chosen solely on the basis of stellar age. Our sample includes three M dwarfs (GJ 182, 799 and 803) which Barrado y Navascués et al. (1999) identified as the youngest of the nearby $\mathrm{M}$ dwarfs, based on the stars' pre-main sequence location on the color-magnitude diagram. The majority of debris disks from IR studies have been identified around A, F, and G-type dwarfs, simply due to observational sensitivity bias (e.g., compilations by Greaves \& Wyatt 2003 and Zuckerman \& Song 2003). The loss processes for circumstellar dust depend on the host star luminosity and gravity. Therefore, studying debris disks over a range of stellar masses may also advance our understanding of this phenomenon.

\section{OBSERVATIONS}

Sub-mm observations were made from December 2002 to November 2003 using the SCUBA bolometer array (Holland et al. 1999) at the 15-m James Clerk Maxwell Telescope (JCMT) on Mauna Kea, Hawaii. ${ }^{4}$ Zenith optical depths ranged from 0.25 to 0.35 at $850 \mu \mathrm{m}$. GJ 803 was first observed on October 5, 2003 and well-detected at $850 \mu \mathrm{m}$; additional $450 \mu \mathrm{m}$ data were obtained on November 12-13, 2003 in dry, stable conditions, with $450 \mu \mathrm{m}$ zenith optical depths ranging from 1.37 to 1.47 . We used the photometry mode to maximize observing efficiency, with integration times ranging from 18 to $96 \mathrm{~min}$ on-source. The data were reduced using Starlink software and standard reduction techniques (see Jenness et al. 2002). Pointing was checked approximately once per hour and was accurate to better than $2^{\prime \prime}$ rms over each night. Flux calibration factors were derived from observations of Uranus or Neptune when available, but secondary calibrators (CRL 618 or CRL 2688) were also used.

We also observed GJ 803 with SCUBA on November 1213, 2003 in jiggle-mapping mode. The weather was stable during the observations with $850 \mu \mathrm{m}$ zenith optical depths ranging from 0.26 to 0.29 . Pointing was checked every $1-2$ hours using Neptune and was accurate to an rms error of $2^{\prime \prime}$. Flux calibrations were performed by observing Uranus and Neptune at the start of each night and were consistent between the two nights to within $10 \%$. A total of 24 individual maps were made at a range of image rotation angles on the array. GJ 803 was well-detected though the emission is not extended at the signal-to-noise level $(\mathrm{S} / \mathrm{N} \approx 8)$ and angular resolution $\left(14^{\prime \prime}=140 \mathrm{AU}\right)$ of the map. The integrated $850 \mu \mathrm{m}$ flux in the map is consistent with earlier measurements made in photometry mode. The emission is also consistent with being centered on the star given the pointing uncertainties.

A sensitive search for $\mathrm{CO} J=3-2$ line emission from GJ 803 was conducted with the B3 dual-channel heterodyne receiver and digital autocorrelating spectrometer (DAS) backend on the JCMT on November 5, 2003. Observations were performed in beam-switching mode with a $60^{\prime \prime}$ throw. Weather conditions were good, and the system temperature ranged from 400 to $450 \mathrm{~K}$ over the elevation range of the observations $\left(30-40^{\circ}\right)$. A total of $200 \mathrm{~min}$ of on-source integration was obtained. No signal was detected to a $1 \sigma$ level of $13 \mathrm{mK}$ per $1 \mathrm{~km} \mathrm{~s}^{-1}$ velocity channel.

\section{RESULTS}

Table 1 presents our sample and SCUBA photometry. We detected GJ 182 and GJ 803 at $850 \mu \mathrm{m}$, with a possible (formally $2 \sigma$ ) detection of GJ 803 at $450 \mu \mathrm{m}$. GJ 803 is also detected at $60 \mu \mathrm{m}$ in the IRAS Faint Source Cata$\log$ (FSC) (Mathioudakis \& Doyle 1991; Song et al. 2002). In addition, we searched for all our targets in the IRAS dataset using the SCANPI algorithm (Helou et al. 1988), via the Infrared Processing and Analysis Center (IPAC) web site. This search revealed $25 \mu \mathrm{m}$ (color-corrected) fluxes of $65 \pm 14$ and $157 \pm 26 \mathrm{mJy}$ for GJ 182 and GJ 803, respectively, which were not included in the FSC.

For GJ 803, Figure 1 shows the 60 and $850 \mu \mathrm{m}$ excess emission modeled with a single temperature fit. (The $25 \mu \mathrm{m}$ flux is consistent within $1 \sigma$ with being purely photospheric in origin.) For the fit, we use a modified blackbody, where the emissivity is constant for $\lambda<100 \mu \mathrm{m}$ and follows $\lambda^{-\beta}$ for longer wavelengths. The spectral energy distributions (SEDs) of debris disks with more extensive multi-wavelength data are well described by this prescription, with $\beta=1.1$ to 0.5 (Dent et al. 2000). For this range of $\beta$, the GJ 803 data are fit with $T=35-45 \mathrm{~K}$, with $T=40 \pm 2 \mathrm{~K}$ for a nominal $\beta=0.8$, where the uncertainty is the formal $1 \sigma$ error in the fit. (Fitting a pure blackbody to the data gives $62 \pm 5 \mathrm{~K}$.) Note that the IRAS nondetection at $100 \mu \mathrm{m}$ is a good match to $\beta=0.8$ and rules out $\beta \gtrsim 1$. With slightly greater sensitivity, IRAS should have detected this source. In addition, the marginal detection at $450 \mu \mathrm{m}$ agrees well with the $\beta=0.8 \mathrm{fit}$, but is inconsistent with a pure blackbody. The integrated fractional dust luminosity $L_{\text {dust }} / L_{\text {star }}$ is $6.1 \times 10^{-4}$ for the nominal $\beta=0.8, T=40 \mathrm{~K}$ model.

For GJ 182, the excess emission at $25 \mu \mathrm{m}$ and $850 \mu \mathrm{m}$ cannot be fit with a single modified blackbody that also

\footnotetext{
4 The JCMT is operated by the Joint Astronomy Centre in Hilo, Hawaii on behalf of the parent organizations Particle Physics and Astronomy
} Research Council in the United Kingdom, the National Research Council of Canada and The Netherlands Organization for Scientific Research. 
satisfies the IRAS non-detections. Ignoring the $25 \mu \mathrm{m}$ flux for the moment, a $40 \mathrm{~K}, \beta=0.8$ modified blackbody, like that which fits the GJ 803 SED, is consistent with the 60, 100 , and $850 \mu \mathrm{m}$ data (Figure 1). A higher dust temperature would violate the $60 \mu \mathrm{m}$ upper limit. Of course, a lower dust temperature would also be consistent with the $850 \mu \mathrm{m}$ detection. Firmer constraints on the dust temperatures await more sensitive IR/sub-mm measurements.

The mid-IR SEDs are sensitive to the presence of warm dust in the inner disk regions. GJ 182 shows a strong $25 \mu \mathrm{m}$ excess (but see $\S 4.2$ ). If we add a $150-200 \mathrm{~K}$ component which emits as a $\beta=0.8$ modified blackbody, a dust mass of $(4-12) \times 10^{-5} M_{\oplus}$ is needed to account for the $25 \mu \mathrm{m}$ excess, or $0.3-0.9 \%$ of the $\mathrm{T}=40 \mathrm{~K}$ dust component which satisfies the $850 \mu \mathrm{m}$ flux (Figure 1). For GJ 803, the large mid-IR dip indicates an absence of warm dust in the inner regions. If we add a warm component which emits as a $\beta=0.8$ modified blackbody, only a very small amount of $150-200 \mathrm{~K}$ dust is permitted, since the IRAS $25 \mu \mathrm{m}$ flux is consistent with being photospheric. Taking the marginal $25 \mu \mathrm{m}$ excess at face value gives a warm dust mass of $(3-9) \times 10^{-6} M_{\oplus}$, or $0.03-0.08 \%$ of the $\mathrm{T}=40 \mathrm{~K}$ dust component.

For the entire sample, we compute dust masses from the $850 \mu \mathrm{m}$ fluxes in the standard fashion, assuming optically thin emission characterized by a single temperature:

$$
M_{d u s t}=\frac{F_{\nu} d^{2}}{\kappa_{\nu} B_{\nu}(T)}
$$

where $F_{\nu}$ is the flux density, $d$ is distance, $\kappa_{\nu}$ is the dust opacity at the observing frequency, and $B_{\nu}$ is the Planck function for a dust temperature $T$. We adopt a dust opacity of $1.7 \mathrm{~cm}^{2} \mathrm{~g}^{-1}$, in agreement with past studies (Zuckerman \& Becklin 1993; Greaves et al. 1998; Holland et al. 1998; Sylvester et al. 2001; Wyatt et al. 2003; Williams et al. 2003; Sheret et al. 2003). This value is on the upper end of the $0.4-1.7 \mathrm{~cm}^{2} \mathrm{~g}^{-1}$ range discussed by Pollack et al. (1994). For non-detections, we use $3 \sigma$ upper limits on the $850 \mu \mathrm{m}$ flux and assume $T=30-100 \mathrm{~K}$, in agreement with previously detected debris disks (Dent et al. 2000). For GJ 803, we adopt the $40 \mathrm{~K}$ temperature from the SED fitting. The calculated dust masses are presented in Table 1.

It is unlikely that our detections are due to background galaxies. Scott et al. (2002) estimate a surface density of about 500 objects per square degree brighter than an $850 \mu \mathrm{m}$ flux of $6 \mathrm{mJy}$, about the $3 \sigma$ sensitivity of our survey. The probability of any background objects to be within an angular distance $\theta$ of a target is $1-\exp \left(-\pi \theta^{2} \Sigma\right)$, where $\Sigma$ is the surface density of background objects above a specified flux level. We compute the ensemble probability of detecting any background objects in SCUBA's central bolometer for our entire sample of 8 objects, accounting for the fact that we mapped GJ 803. This gives a $4 \%$ probability that background sources would produce at least one detection in our survey.

The GJ 803 system appears to have very little molecular gas. The CO $3-2$ intensity is $-45 \pm 49 \mathrm{mK} \mathrm{km} \mathrm{s}^{-1}$ integrated over $\pm 7 \mathrm{~km} \mathrm{~s}^{-1}$ about the stellar velocity. (This velocity range corresponds to the maximum orbital speed for a disk viewed at an inclination of $80^{\circ}$ with the inner disk edge of $17 \mathrm{AU}$ derived from the SED fit.) Assuming the gas is optically thin and in thermal equilibrium with
$40 \mathrm{~K}$ dust, the $3 \sigma$ upper limit implies a CO column density of $6.2 \times 10^{13} \mathrm{~cm}^{-2}$. Temperatures as warm as $\approx 150 \mathrm{~K}$, e.g., if the gas were located in the inner few AU, would give inferred CO column densities of a factor of 2 larger. Determining an upper limit on the total gas mass is uncertain, since this is dominated by $\mathrm{H}_{2}$ and the $\mathrm{H}_{2}$ to $\mathrm{CO}$ conversion is highly uncertain. CO may freeze-out onto grains, making it a poor tracer of the total disk mass. Also, photoionization may affect the $\mathrm{H}_{2}$ to $\mathrm{CO}$ conversion factor. To attempt to account for photoionization by the interstellar UV field, we refer to calculations by van Dishoeck \& Black (1988), slightly extrapolated to lower CO column densities, and adopt an $\mathrm{H}_{2}$ to $\mathrm{CO}$ abundance ratio of $10^{-7}$ (cf., normal abundance ratio of $10^{-4}$ ). This results in an $\mathrm{H}_{2}$ mass of $1.3 M_{\oplus}$, if we ignore the possibility of $\mathrm{CO}$ freeze-out. Overall, the gas non-detection limits for GJ 803 are comparable to those for older solar-type stars (Dent et al. 1995; Greaves et al. 2000a) and rules out the possibility of GJ 803 having a gas-rich disk comparable to those detected around younger stars (Zuckerman et al. 1995; Greaves et al. 2000b).

\section{DISCUSSION}

\subsection{Sub-Millimeter Evolution of Debris Disks}

Figure 2 summarizes the known debris disk mass estimates based on sub-mm observations from our work and the published literature. Our SCUBA survey adds a significant number of stars of $\approx 10-50$ Myr. Our raw $850 \mu \mathrm{m}$ sensitivity (median rms of $1.9 \mathrm{mJy}$ ) is poorer than the Wyatt et al. (2003) survey of Lindroos binaries (median rms of $1.6 \mathrm{mJy}$ ). But given the much closer distances of our targets, our dust mass sensitivity is better by around an order of magnitude. In particular, Figure 2 shows the following:

1. Compared to primordial disks around $T$ Tauri and Herbig Ae/Be stars which have dust masses of $\approx 10$ $300 M_{\oplus}$ (e.g. Osterloh \& Beckwith 1995; Natta et al. 2000), the dust masses for the detected young stars are $\sim 10^{3}$ smaller. This points to very rapid evolution of the circumstellar dust mass within the first $\sim 10 \mathrm{Myr}$, likely arising from grain removal (e.g., via accretion onto the central star) and/or grain growth into larger bodies, which would greatly diminish the sub-mm/mm emission.

2. The upper envelope for disk masses around A stars exceeds that for later-type stars. This may reflect the distribution of primordial disk masses; for $\mathrm{T}$ Tauri stars a similar trend is seen, where the upper envelope of primordial disk masses is larger for more massive stars (Natta 2003).

3. For stars of $\approx 10-100$ Myr old, nearly all the detected disks at a given spectral type (i.e., stellar mass) are less massive than the upper limits on non-detections. This highlights the fact that existing sub-mm measurements are only sensitive to the most massive of the young debris disks.

4. The apparent correlation of dust mass with stellar age arises from observational bias: the young stars detected in the sub-mm are at larger distances than 
the old stars. However, the decline in the upper envelope of disk masses with age is probably a real effect. While there are no published sub-mm data in the upper right of Figure 2 (i.e., old stars with massive disks), the existing sub-mm detections of nearby, old stars come from sources known to possess large IR excesses in IRAS and ISO data. Any old stars with even more massive disks would have been detected by these IR surveys, unless the dust was unprecedentedly cold. Based on the data in Figure 2, the masses of sub-mm detected disks evolve roughly as $M_{d u s t} \sim t^{-0.5}$ to $t^{-1}$, with a simple unweighted fit giving $M_{d u s t} \propto t^{-(0.7 \pm 0.2)}$.

\subsection{Debris Disks and Planets around Low-Mass Stars}

GJ 182 and GJ 803 are the first M-star debris disks detected at sub-millimeter wavelengths. ${ }^{5}$ The dust lifetime is short compared to the stars' ages (e.g. Backman \& Paresce 1993). Therefore, the dust must be replenished by collisions between planetesimals, either in situ or else from regions farther out. Note that for these low luminosity stars $\left(0.1-0.2 L_{\odot}\right)$, radiation pressure on the grains is negligible compared to the star's gravity, unlike debris disks around A stars, e.g., $\beta$ Pic where micron-sized and smaller grains are expelled (Artymowicz 1988).

Our work shows that most debris disks around low-mass stars probably lie at or below the sensitivity limits of the IRAS survey and JCMT/SCUBA observations. GJ 182 is just at the practical limit for IRAS and SCUBA detection. GJ 803 is the nearest object in our sample, and among the nearest known young stars, which makes detection of its very tenuous dust mass feasible. The one undetected M dwarf in our sample, GJ 799, is just as close and young as GJ 803. GJ 799 is known to be a $3^{\prime \prime}$ binary of comparable magnitude (Mason et al. 2001), which may impact its dust content. In addition, its M4.5 spectral type corresponds to $M_{\star} \approx 0.10-0.15 M_{\odot}$, about $3-4 \times$ smaller than for GJ 182 and GJ 803 (e.g., see models presented in Hillenbrand 1997 and Luhman 1999). Hence, the nondetection may simply be due to insufficient sensitivity; our JCMT data for GJ 799 would have to be $\approx 4 \times$ deeper in order to reach the same $M_{d u s t} / M_{\text {star }}$ as for the two detected $\mathrm{M}$ dwarfs. Primordial disks are common around low mass stars (Haisch et al. 2001a) and even brown dwarfs (Liu et al. 2003) at ages of a few Myr, and these systems may very well generate debris disks as they age. The Spitzer Space Telescope will offer the requisite sensitivity for detecting and studying such disks.

For GJ 182, the $25 \mu \mathrm{m}$ excess suggests that dust resides in the inner regions of its disk: $150-200 \mathrm{~K}$ blackbody grains would lie at 1-2 AU from the star. Given the sparse sampling of the current SED, one cannot determine if the dust distribution is continuous throughout the disk, or if there is a gap in the middle regions of the disk (e.g., from planet formation) between the $25 \mu \mathrm{m}$ and $850 \mu \mathrm{m}$ emitting dust. However, as shown by Song et al. (2002), single band excesses near the sensitivity limit of the IRAS catalog may not be reliable. Though the SCANPI detection of GJ 182 has $\mathrm{S} / \mathrm{N}=4.6$, higher than the $\mathrm{S} / \mathrm{N}=2-4$ ob- jects examined by Song et al., for now we treat the $25 \mu \mathrm{m}$ excess of GJ 182 as tentative. More detailed SED measurements, in particular with Spitzer, would be invaluable to characterize this star's disk.

The absence of warm dust in the inner regions of GJ 803 (and perhaps GJ 182, if the $25 \mu \mathrm{m}$ detection is spurious) is naturally explained by an unseen inner companion. The $40 \mathrm{~K}$ temperature characteristic of the SED is too cold to be explained by sublimation or melting of icy grains $(\approx 100-170 \mathrm{~K})$. Without an inner companion, the effect of Poynting-Robertson drag would cause grains to spiral inward and produce significant mid-IR emission. Such inner holes are typical for debris disk systems. Indeed, the few debris disks with resolved dust emission show morphologies highly suggestive of the dynamical influence of an unseen companion at mean-motion resonances (Holland et al. 1998; Greaves et al. 1998; Ozernoy et al. 2000; Koerner et al. 2001; Wilner et al. 2002; Quillen \& Thorndike 2002; Wahhaj et al. 2003; Kuchner \& Holman 2003; Thommes \& Lissauer 2003).

The $40 \mathrm{~K}$ characteristic temperature from the SED fitting means that blackbody grains around GJ 803 would be at a distance of $17 \mathrm{AU}$, or an impressively large angular separation of $1.7^{\prime \prime}$. (For GJ 182, 17 AU corresponds to only $0.6^{\prime \prime}$.) This provides only a rough guide as to the angular separation of any interior companion, since it is difficult to use the SEDs of debris disks to predict the spatial dust distribution (Sheret et al. 2003). Shallow IR adaptive optics imaging from the Keck Observatory with $0.05^{\prime \prime}$ resolution shows no stellar or massive brown dwarf companion, suggesting that an even lower mass companion is present. To date, GJ 876 is the only $\mathrm{M}$ dwarf known to have extrasolar planets (Marcy et al. 1998, 2001); its two planets have semi-major axes of 0.13 and $0.21 \mathrm{AU}$.

Thus the GJ 803 inner disk hole may indicate that planets can exist at larger separations around $M$ dwarfs than known so far and that such planets can form within $\approx 10 \mathrm{Myr}$. Furthermore, given the very young age of the system, a planetary companion is predicted to have significant thermal emission (e.g. Burrows et al. 1997) and hence might be detectable with deeper AO imaging. M dwarfs are by far the most numerous type of star, so a better understanding of their planet-bearing frequency is needed for a complete census of the extrasolar planet population.

Follow-up coronagraphic imaging by Kalas et al. (2004) finds that GJ 803 has a large, nearly edge-on disk seen in scattered optical light. This disk is detected from 5$21^{\prime \prime}$ (50-210 AU), though the inner and outer extent are not well-constrained. Our sub-mm imaging finds that the $850 \mu \mathrm{m}$ dust emission is unresolved. This is entirely compatible with the large optical disk, given that the grains responsible for scattering may be very cold and/or small, and hence poor sub-mm emitters. Deeper sub-mm mapping is needed for stronger constraints on any extended emission. In general, the proximity and youthfulness of GJ 803, combined with the high sub-mm and optical detectability of its disk, make this system an excellent opportunity to study disk evolution, and perhaps planet for-

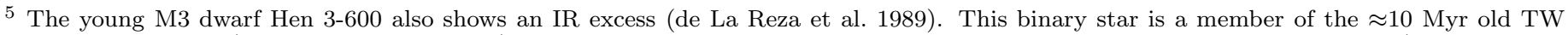

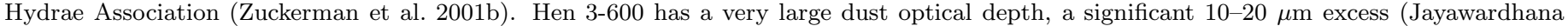

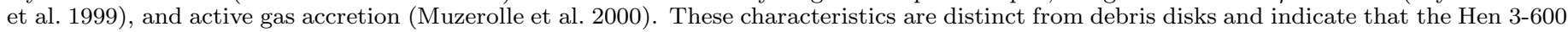
disk is primordial or else in a transitional state. 
mation, in great detail.

The onset and duration of the debris disk phenomenon remain open questions. As discussed by Greaves \& Wyatt (2003), the disk masses might slowly decay over time, or perhaps the mass distribution is bimodal, reflecting "on" and "off" states for detectability. The GJ 803 debris disk offers some insight into this issue. Using the Hipparcos catalog, which is nearly complete for early-type $M$ dwarfs (M0-M2) within 10 pc, Song et al. (2002) found GJ 803 was the only object out of a sample of $152 \mathrm{M}$ dwarfs to possess an IR excess in the IRAS FSC. This is not due to detection bias, as there are many early-type $M$ dwarfs which have closer distances than GJ 803. GJ 803 is also known to be among the youngest of the nearby $\mathrm{M}$ dwarfs (Barrado y Navascués et al. 1999; Zuckerman et al. 2001a). If the appearance of debris disks was an intermittent event, Song et al. should have detected older M dwarfs with IR excesses at both 60 and $100 \mu \mathrm{m}$, if their disks were like the GJ 803 one. Either these older M dwarfs do not have disks, or their disks are much less massive than GJ 803. Therefore, at least for the case of M dwarfs, the GJ 803 system suggests that the time evolution of debris disks is driven primarily by age, with the youngest stars having the largest disk masses.

We thank Tom Chester for discussions about IRAS SCANPI processing. This research has made use of the NASA/IPAC Infrared Science Archive (IRSA), the SIMBAD database, and the Washington Double Star Catalog maintained at the U.S. Naval Observatory. We are grateful for support from NASA grant HST-HF-01152.01 (MCL), NSF grant AST-0228963 (BCM), NSF grant AST-0324328 (JPW), and NASA Origins Program grant NAG5-11769 (PGK).

\section{REFERENCES}

Allard, F., Hauschildt, P. H., Alexander, D. R., Tamanai, A., \& Schweitzer, A. 2001, ApJ, 556, 357

Allard, F., Hauschildt, P. H., \& Schwenke, D. 2000, ApJ, 540, 1005 Artymowicz, P. 1988, ApJ, 335, L79

Aumann, H. H., Beichman, C. A., Gillett, F. C., de Jong, T., Houck, J. R., Low, F. J., Neugebauer, G., Walker, R. G., \& Wesselius, P. R. 1984, ApJ, 278, L23

Backman, D. E. \& Paresce, F. 1993, in Protostars and Planets III, 1253

Barrado y Navascués, D., Stauffer, J. R., Song, I., \& Caillault, J.-P. 1999, ApJ, 520, L123

Burrows, A., Marley, M., Hubbard, W. B., Lunine, J. I., Guillot, T., Saumon, D., Freedman, R., Sudarsky, D., \& Sharp, C. 1997, ApJ, 491,856

de La Reza, R., Torres, C. A. O., Quast, G., Castilho, B. V., \& Vieira, G. L. 1989, ApJ, 343, L61

Decin, G., Dominik, C., Malfait, K., Mayor, M., \& Waelkens, C. 2000, A\&A, 357, 533

Dent, W. R. F., Greaves, J. S., Mannings, V., Coulson, I. M., \& Walther, D. M. 1995, MNRAS, 277, L25

Dent, W. R. F., Walker, H. J., Holland, W. S., \& Greaves, J. S. 2000, MNRAS, 314, 702

Fajardo-Acosta, S. B., Stencel, R. E., Backman, D. E., \& Thakur, N. 1999, ApJ, 520, 215

Favata, F., Micela, G., Sciortino, S., \& D’Antona, F. 1998, A\&A, 335,218

Gahm, G. F., Zinnecker, H., Pallavicini, R., \& Pasquini, L. 1994, A\&A, 282, 123

Gaidos, E. J. 1999, ApJ, 510, L131

Greaves, J. S., Coulson, I. M., \& Holland, W. S. 2000a, MNRAS, 312, L1

Greaves, J. S., Holland, W. S., Moriarty-Schieven, G., Jenness, T., Dent, W. R. F., Zuckerman, B., McCarthy, C., Webb, R. A., Butner, H. M., Gear, W. K., \& Walker, H. J. 1998, ApJ, 506, L133

Greaves, J. S., Mannings, V., \& Holland, W. S. 2000b, Icarus, 143, 155

Greaves, J. S. \& Wyatt, M. C. 2003, MNRAS, 345, 1212

Habing, H. J., Dominik, C., Jourdain de Muizon, M., Kessler, M. F., Laureijs, R. J., Leech, K., Metcalfe, L., Salama, A., Siebenmorgen, R., \& Trams, N. 1999, Nature, 401, 456

Habing, H. J., Dominik, C., Jourdain de Muizon, M., Laureijs, R. J., Kessler, M. F., Leech, K., Metcalfe, L., Salama, A., Siebenmorgen, R., Trams, N., \& Bouchet, P. 2001, A\&A, 365, 545

Haisch, K. E., Lada, E. A., \& Lada, C. J. 2001a, AJ, 121, 2065

-. 2001b, ApJ, 553, L153

Helou, G., Khan, I. R., Malek, L., \& Boehmer, L. 1988, ApJS, 68, 151

Hillenbrand, L. A. 1997, AJ, 113, 1733

Holland, W. S., Greaves, J. S., Dent, W. R. F., Wyatt, M. C., Zuckerman, B., Webb, R. A., McCarthy, C., Coulson, I. M., Robson, E. I., \& Gear, W. K. 2003, ApJ, 582, 1141

Holland, W. S., Greaves, J. S., Zuckerman, B., Webb, R. A., McCarthy, C., Coulson, I. M., Walther, D. M., Dent, W. R. F., Gear, W. K., \& Robson, I. 1998, Nature, 392, 788
Holland, W. S., Robson, E. I., Gear, W. K., Cunningham, C. R., Lightfoot, J. F., Jenness, T., Ivison, R. J., Stevens, J. A., Ade, P. A. R., Griffin, M. J., Duncan, W. D., Murphy, J. A., \& Naylor, D. A. 1999, MNRAS, 303, 659

Holmes, E. K., Butner, H. M., Fajardo-Acosta, S. B., \& Rebull, L. M. 2003, AJ, 125, 3334

Jayawardhana, R., Hartmann, L., Fazio, G., Fisher, R. S., Telesco, C. M., \& Piña, R. K. 1999, ApJ, 520, L41

Jeffries, R. D. 1995, MNRAS, 273, 559

Jenness, T., Stevens, J. A., Archibald, E. N., Economou, F., Jessop, N. E., \& Robson, E. I. 2002, MNRAS, 336, 14

Jewitt, D. C. 1994, AJ, 108, 661

Kalas, P., Liu, M. C., \& Matthews, B. C. 2004, Science, in press

Koerner, D. W., Sargent, A. I., \& Ostroff, N. A. 2001, ApJ, 560, L181

Kuchner, M. J. \& Holman, M. J. 2003, ApJ, 588, 1110

Laureijs, R. J., Jourdain de Muizon, M., Leech, K., Siebenmorgen, R., Dominik, C., Habing, H. J., Trams, N., \& Kessler, M. F. 2002, A\&A, 387, 285

Lindroos, K. P. 1986, A\&A, 156, 223

Liu, M. C., Najita, J., \& Tokunaga, A. T. 2003, ApJ, 585, 372

Luhman, K. L. 1999, ApJ, 525, 466

Marcy, G. W., Butler, R. P., Fischer, D., Vogt, S. S., Lissauer, J. J., \& Rivera, E. J. 2001, ApJ, 556, 296

Marcy, G. W., Butler, R. P., Vogt, S. S., Fischer, D., \& Lissauer, J. J. 1998, ApJ, 505, L147

Mason, B. D., Wycoff, G. L., Hartkopf, W. I., Douglass, G. G., \& Worley, C. E. 2001, AJ, 122, 3466

Mathioudakis, M. \& Doyle, J. G. 1991, A\&A, 244, 433

Montes, D., López-Santiago, J., Gálvez, M. C., Fernández-Figueroa, M. J., De Castro, E., \& Cornide, M. 2001, MNRAS, 328, 45

Muzerolle, J., Briceño, C., Calvet, N., Hartmann, L., Hillenbrand, L., \& Gullbring, E. 2000, ApJ, 545, L141

Natta, A. 2003, in Debris Disks and the Formation of Planets: a Symposium in Memory of Fred Gillett, ed. D. Backman \& L. Caroff, ASP. Conf. Series, in press (astro-ph/0304184)

Natta, A., Grinin, V., \& Mannings, V. 2000, Protostars and Planets IV, 559

Osterloh, M. \& Beckwith, S. V. W. 1995, ApJ, 439, 288

Ozernoy, L. M., Gorkavyi, N. N., Mather, J. C., \& Taidakova, T. A. 2000, ApJ, 537, L147

Plets, H. \& Vynckier, C. 1999, A\&A, 343, 496

Pollack, J. B., Hollenbach, D., Beckwith, S., Simonelli, D. P., Roush, T., \& Fong, W. 1994, ApJ, 421, 615

Quillen, A. C. \& Thorndike, S. 2002, ApJ, 578, L149

Ray, T. P., Sargent, A. I., Beckwith, S. V. W., Koresko, C., \& Kelly, P. 1995, ApJ, 440, L89

Scott, S. E., Fox, M. J., Dunlop, J. S., Serjeant, S., Peacock, J. A., Ivison, R. J., Oliver, S., Mann, R. G., Lawrence, A., Efstathiou, A., Rowan-Robinson, M., Hughes, D. H., Archibald, E. N., Blain, A., \& Longair, M. 2002, MNRAS, 331, 817

Sheret, I., Dent, W. R. F., \& Wyatt, M. C. 2003, MNRAS, in press (astro-ph/0311593)

Silverstone, M. D. 2000, Ph.D. Thesis

Song, I., Weinberger, A. J., Becklin, E. E., Zuckerman, B., \& Chen, C. $2002, \mathrm{AJ}, 124,514$

Spangler, C., Sargent, A. I., Silverstone, M. D., Becklin, E. E., \& Zuckerman, B. 2001, ApJ, 555, 932 
Strom, S. E., Edwards, S., \& Skrutskie, M. F. 1993, in Protostars and Planets III, 837-866

Sylvester, R. J., Dunkin, S. K., \& Barlow, M. J. 2001, MNRAS, 327, 133

Sylvester, R. J. \& Mannings, V. 2000, MNRAS, 313, 73

Sylvester, R. J., Skinner, C. J., Barlow, M. J., \& Mannings, V. 1996, MNRAS, 279, 915

Thommes, E. W. \& Lissauer, J. J. 2003, ApJ, 597, 566

van Dishoeck, E. F. \& Black, J. H. 1988, ApJ, 334, 771

Wadhwa, M. \& Russell, S. S. 2000, Protostars and Planets IV, 995

Wahhaj, Z., Koerner, D. W., Ressler, M. E., Werner, M. W., Backman, D. E., \& Sargent, A. I. 2003, ApJ, 584, L27

Williams, J. P., Najita, J., Liu, M. C., Bottinelli, S., Carpenter, J. M., Hillenbrand, L. A., Meyer, M. R., \& Soderblom, D. R. 2003, ApJ, in press (astro-ph/0311583)
Wilner, D. J., Holman, M. J., Kuchner, M. J., \& Ho, P. T. P. 2002, ApJ, 569, L115

Wyatt, M. C., Dent, W. R. F., \& Greaves, J. S. 2003, MNRAS, 342, 876

Zuckerman, B. 2001, ARA\&A, 39, 549

Zuckerman, B. \& Becklin, E. E. 1993, ApJ, 414, 793

Zuckerman, B., Forveille, T., \& Kastner, J. H. 1995, Nature, 373, 494

Zuckerman, B. \& Song, I. 2003, ApJ, in press (astro-ph/0311546)

Zuckerman, B., Song, I., Bessell, M. S., \& Webb, R. A. 2001a, ApJ, 562, L87

Zuckerman, B., Webb, R. A., Schwartz, M., \& Becklin, E. E. 2001b, ApJ, 549, L233 

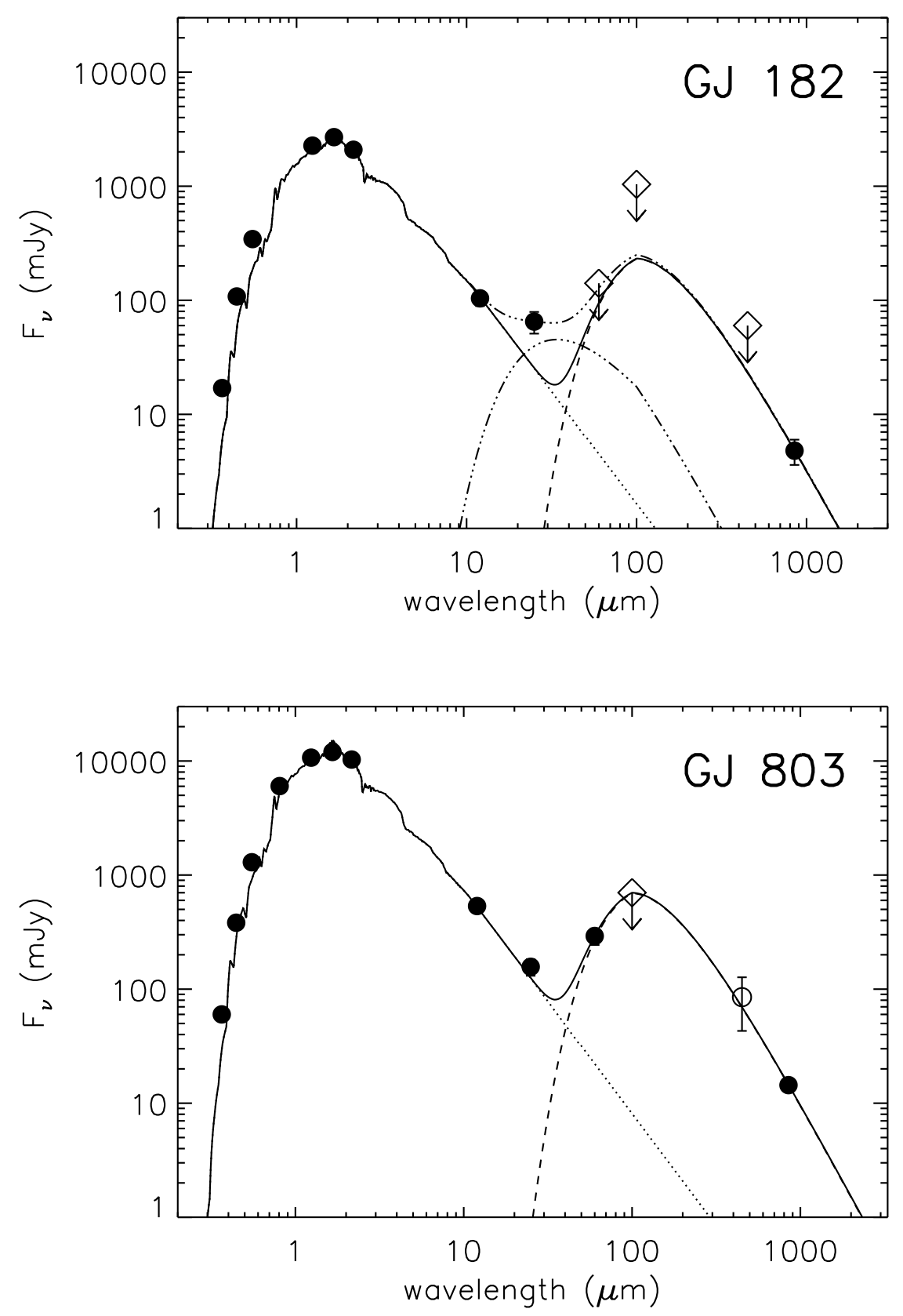

FIg. 1.- Observed SEDs of our stars detected by SCUBA. Detections are plotted as filled circles and non-detections as open symbols with arrows. The possible detection of GJ 803 at $450 \mu \mathrm{m}$ is shown as an open circle. Error bars are shown when they are larger than the symbol size. The data are compared to the sum of a theoretical stellar spectrum (dotted line) and a modified blackbody with $T=40 \mathrm{~K}$ and $\beta=0.8$ which fits the $\geq 60 \mu \mathrm{m}$ detections (dashed line). For GJ 182, we add a $150 \mathrm{~K}$ component to account for the possible $25 \mu \mathrm{m}$ excess (dotted-dashed line). See text for details (§ 3 and $\S 4)$. The optical data $(0.3-0.8 \mu \mathrm{m})$ are from SIMBAD and the Hipparcos catalog. The IR data $(1-100 \mu \mathrm{m})$ are from the 2MASS catalog and IRAS color-corrected Faint Source Catalog and SCANPI photometry. The sub-mm data $(450$ and $850 \mu \mathrm{m})$ are from this paper. The stellar spectra come from the NextGen models by Allard et al. $(2000,2001)$ and are normalized to the observed $K$-band magnitude. 


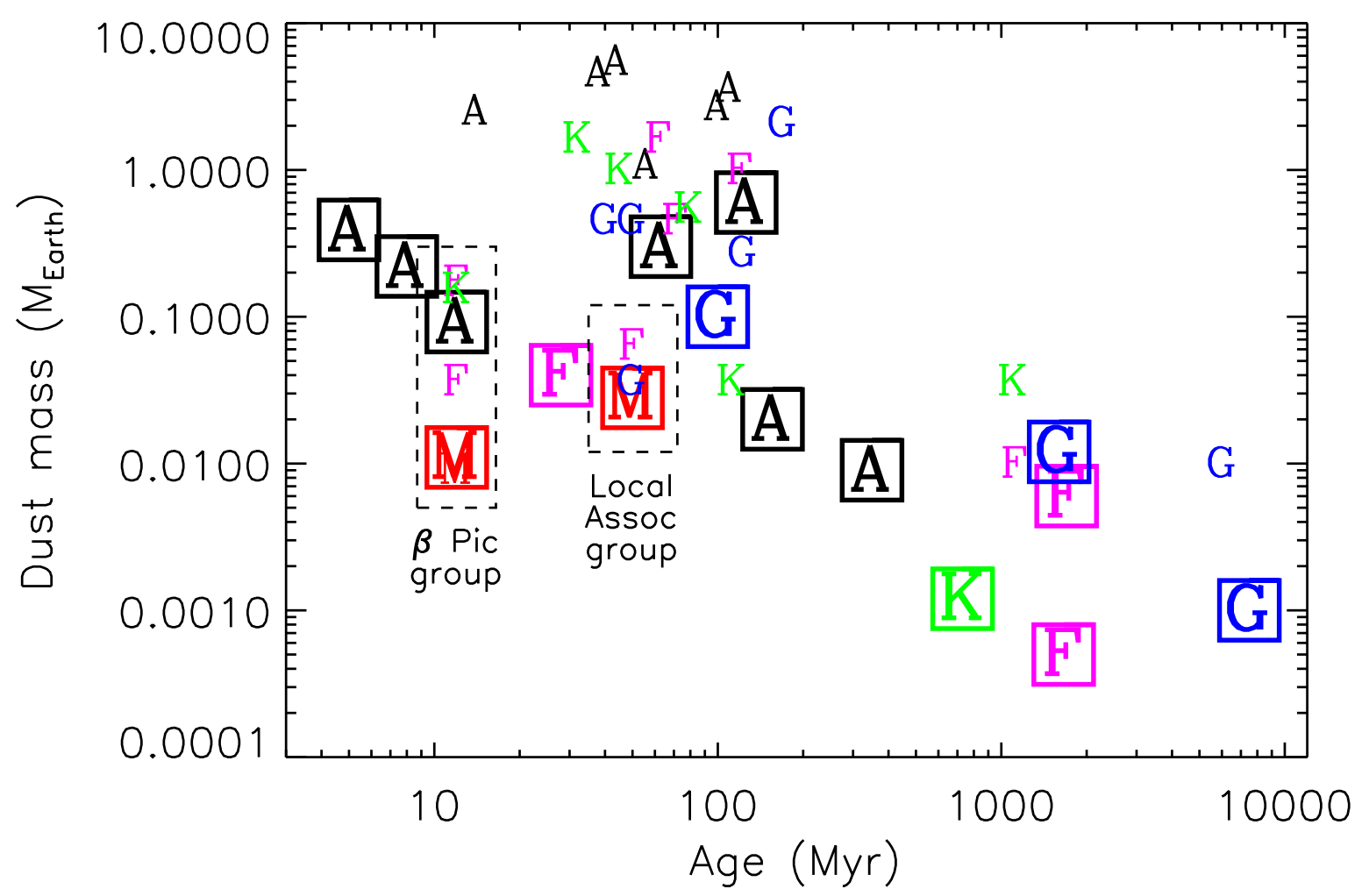

FIG. 2.- Debris disks with dust masses derived from sub-millimeter data. Detections are shown in boxes, where the letters indicate the spectral type. GJ 182 and GJ 803 are the M dwarfs detected in this paper. Non-detections are shown as smaller letters without boxes, assuming a dust temperature of $30 \mathrm{~K}$. The GJ 799 non-detection is obscured behind the M dwarf detection of GJ 803 at 12 Myr. In addition to this paper, data come from compilations by Wyatt et al. (2003) and Sheret et al. (2003) along with the young G star debris disk from Williams et al. (2003). The quoted age uncertainties range from $\approx 25 \%$ to a factor of $\approx 2$. 
TABLE 1

JCMT/SCUBA OBSERVATIONS

\begin{tabular}{|c|c|c|c|c|c|}
\hline Object & $\operatorname{SpT}^{(a)}$ & $\begin{array}{c}d^{(a)} \\
(\mathrm{pc})\end{array}$ & $\begin{array}{c}\lambda \\
(\mu \mathrm{m})\end{array}$ & $\begin{array}{c}F_{\nu}^{(b)} \\
(\mathrm{mJy})\end{array}$ & $\begin{array}{l}M_{d u s t}{ }^{(b)} \\
\left(M_{\oplus}\right)\end{array}$ \\
\hline \multicolumn{6}{|l|}{$\beta$ Pic Group $\left(12_{-4}^{+8} \mathrm{Myr}\right)$} \\
\hline HD $35850^{(c)}$ & F7V & $26.8 \pm 0.6$ & 850 & $<4.5$ & $<0.01-0.04$ \\
\hline HD 199143 & F8V & $47.7 \pm 2.3$ & 850 & $<7.5$ & $<0.05-0.19$ \\
\hline HD 358623 (AZ Cap) & $\mathrm{K} 7 \mathrm{Ve}$ & $47.7 \pm 2.3$ & 850 & $<6.9$ & $<0.04-0.17$ \\
\hline GJ 803 (AU Mic) & M1e & $9.94 \pm 0.13$ & 850 & $14.4 \pm 1.8$ & 0.011 \\
\hline GJ 799 (AT Mic) & M4.5e & $10.2 \pm 0.5$ & $\begin{array}{l}450 \\
850\end{array}$ & $\begin{array}{c}85 \pm 42 \\
<9.0\end{array}$ & $<0.003-0.010$ \\
\hline \multicolumn{6}{|c|}{ Local Association Group $\left(50_{-30}^{+100} \mathrm{Myr}\right)$} \\
\hline EK Dra (HD 129333) & F8 & $33.9 \pm 0.7$ & 850 & $<5.7$ & $<0.02-0.07$ \\
\hline HD 77407 & G0 & $30.1 \pm 0.8$ & 850 & $<4.5$ & $<0.01-0.04$ \\
\hline GJ $182^{(d)}$ & $\mathrm{M} 0.5 \mathrm{Ve}$ & $26.7 \pm 1.8$ & $\begin{array}{l}850 \\
450\end{array}$ & $\begin{array}{l}4.8 \pm 1.2 \\
<60\end{array}$ & $0.007-0.028$ \\
\hline
\end{tabular}

${ }^{\mathrm{a}}$ From SIMBAD and the Hipparcos catalog.

${ }^{\mathrm{b}}$ For non-detections, we use the $3 \sigma$ upper limits on the $850 \mu \mathrm{m}$ flux and assume $T=$ $30-100 \mathrm{~K}$. For GJ 803, the dust mass is computed assuming $T=40 \mathrm{~K}$, derived from the SED fitting.

${ }^{\mathrm{c}}$ Silverstone (2000) report a possible IR excesses based on ISO data, but Decin et al. (2000) suggest the excess may arise from extended cirrus, not from the star itself.

${ }^{\mathrm{d}}$ Montes et al. (2001) show that GJ 182 shares kinematics with the higher mass Local Association stars, which have estimated ages of $\approx 50$ Myr based on stellar activity and Li abundances. However, placing GJ 182 on an HR diagram suggests a younger age of $\approx 10$ 20 Myr (Favata et al. 1998; Barrado y Navascués et al. 1999). 